\title{
Loss of neuropilin1 inhibits liver cancer stem cells population and blocks metastasis in hepatocellular carcinoma via epithelial-mesenchymal transition
}

\author{
Xun $\mathrm{LI}^{1,2, *}$, Yongqiang ZHOU ${ }^{3}$, JinJing $\mathrm{HU}^{2,4}$, Zhongtian BAI ${ }^{1}$, Wenbo MENG ${ }^{1}$, Lei ZHANG ${ }^{1}$, Xiaojing SONG ${ }^{1}$, Yongjian WEI ${ }^{3}$, Jun YAN ${ }^{1}$, Yihua ZHOU ${ }^{3}$ \\ ${ }^{1}$ Department of General Surgery, The First Hospital of Lanzhou University; Cancer Prevention and Treatment Center of Lanzhou University \\ School of Medicine, Lanzhou, China; ${ }^{2}$ Gansu Province Key Laboratory of Biotherapy and Regenerative Medicine, Lanzhou, China; ${ }^{3}$ The First \\ Clinical Medical College of Lanzhou University, Lanzhou, China; ${ }^{4}$ School of Life Science of Lanzhou University, Lanzhou University, Lanzhou, \\ China \\ *Correspondence: lxdr21@126.com
}

Received September 9, 2020 / Accepted November 20, 2020

\begin{abstract}
It is generally believed that the existence of cancer stem cells (CSCs) is related to tumor recurrence and metastasis of hepatocellular carcinoma (HCC). Neuropilin1 (NRP1) is involved in numerous pathophysiological processes of tumor progression, however, whether NRP1 is involved in the regulation of liver CSCs and metastasis of HCC is still unknown. In the present study, we examined the effect of NRP1 on the population of liver CSCs and the metastasis mechanism of HCC. In NRP1 small hairpin RNA (shRNA)-transduced HCC cells, liver CSCs surface markers (CD133+/ EpCAM+/CD13+/ CD44+) expressing cells, which imply the CSCs population, were decreased. Transwell assay and nude mouse liver orthotopic transplantation model confirmed that NRP1 knockdown inhibited HCC cells' migration and lung metastasis. Our data showed that the expression of NRP1 was upregulated in 5 independent cohorts of HCC patients, consequently, high levels of NRP1 correlated with recurrence and poor prognosis in HCC. Mechanism research showed that NRP1 promotes cell spreading through the epithelial-mesenchymal transition (EMT) signaling pathway. In summary, NRP1 enhanced the population of liver CSCs and migration of HCC via EMT, indicating that NRP1 might be a novel target for HCC treatments.
\end{abstract}

Key words: neuropilin1, hepatocellular carcinoma, liver cancer stem cells, tumor metastasis, epithelial-mesenchymal transition

Hepatocellular carcinoma (HCC) is one of the most common primary malignant tumors, causing a huge global health burden. Systematic and reasonable methods of screening, diagnosis, treatment, and follow-up have been formulated. However, morbidity and cancer-specific mortality have not been effectively controlled due to the lack of symptoms in the early stage, hidden onset, primary tumor heterogeneity, and tumor relapse [1].

The infinite differentiation potential and self-renewal properties of cancer stem cells (CSCs) are generally considered to be the causes of HCC cell growth, tumor heterogeneity, resistance to anti-cancer therapies, distant metastasis, recurrence, and treatment failure [2]. The identification of surface molecular targets expressed by CSCs and the investigation of regulatory mechanisms of targeting CSCs biological characteristics are critical to improving response to HCC therapeutic intervention [3]. A variety of markers for identification and isolation of liver cancer stem cells (LCSCs) have been reported, the representative markers are CD13, CD133, CD44, and epithelial cell adhesion molecule (EpCAM) [4].
The expression level of these CSCs surface markers can predict the potential of LCSCs to migrate, self-renew, and differentiate. The prospects for CSCs-targeting therapies are encouraging and optimistic [5], the researches of CSCs regulatory network are particularly important.

Neuropilin1 (NRP1), widely expressing in tumor vasculature, promotes angiogenesis and tumor progression by selectively binding to multiple extracellular signaling ligands [6]. NRP1 is involved in a wide range of pathophysiological processes, its expression and function in nerve cells, tumor cells, and immune cells have been extensively investigated [7]. In gastric cancer [8], breast cancer [9], colorectal cancer [10], oral squamous cell carcinoma [11], and lung cancer [12], elevated NRP1 expression is associated with cancer progression and poor clinical prognosis. NRP1 may be a promising target for cancer immunotherapy. $\mathrm{CD}^{+} \mathrm{T}$ cell-restricted NRP1-deficient mice are significantly more sensitive to PD1 immunotherapy [13]. The high proportion of $\mathrm{NRP}^{-} /^{-}$ regulatory $\mathrm{T}$ cells (Tregs) in the tumor enhances anti-tumor immunity and promotes tumor clearance [14]. During CSCs 
promotes tumor progression and deterioration, angiogenesis is crucial, NRP1 acts as a vascular endothelial growth factor (VEGF) receptor, the regulation of NRP1 on CSCs has been attracting people's attention.

In the current study, we investigated the effect of NRP1 on the level of CD13, CD133, CD44, and EpCAM. The application of HCC cell lines, HCC tumor specimens, and liver orthotopic transplantation models verified that elevated NRP1 boosts migration, recurrence, and metastasis. The clinical prognosis of HCC patients with high expression of NRP1 is poor. Finally, we concluded that the high level of NRP1 elevates the proportion of LCSCs, thus promoting the malignant potential of HCC, and NRP1 may be used as a target for anti-LCSCs therapies.

\section{Patients and methods}

Patients and follow-up. Five independent patient cohorts were recruited as participants, with a total of 372 HCC patients (the cohorts, number of cases, sample source, and detection methods are summarized in Supplementary Table S1). Tumor and matched paracancer tissue were obtained after curative hepatic resections, which were performed at the Zhongshan Hospital of Fudan University during 2001-2010 and all patients gave written informed consent before the study. All cancers were verified histologically. The study was approved by the Hospital Ethics Committee and informed consent was obtained from each patient under Institutional Reviewer Board protocols. The study protocol conforms to the ethical guidelines of the 1975 Declaration of Helsinki as reflected in a priori approval by the institution's human research committee.

Tissue microarrays and immunohistochemistry. Paraffin-embedded tissue samples were obtained from 239 patients. Core samples from representative areas of each tumor were selected for $\mathrm{H} \& \mathrm{E}$ staining. Immunostaining of NRP1 was performed. Briefly, $4 \mu \mathrm{m}$ tissue sections were deparaffinized and treated for antigen retrieval (citrate buffer, pH 6.0). Sections were then incubated (30 min) with rabbit monoclonal antibodies to NRP1 (1:100; Abcam), exposed to 3,3'-diaminobenzidine tetrahydrochloride, and counterstained with hematoxylin. Semi-quantitation was based on the intensity of staining of positive cells as follows: 0$)<5 \%$; 1) $6-35 \%$; 2) $36-70 \%$; 3) $>70 \%$. Slides were assessed by two observers independently.

RNA isolation and qRT-PCR. Total RNA was extracted using TRIzol reagent (Invitrogen, USA) as instructed by the manufacturer. NRP1 expression was analyzed using an ABI7900HT unit (Applied Biosystems, Foster City, USA). All qRT-PCR testing relied on TB Green Premix Ex Taq II (Takara Bio, Shiga, Japan), following the manufacturer's instructions. The primers are shown in Supplementary Table S2. Results were normalized using the endogenous control gene (GAPDH) expression. The relative expression levels of NRP1 were analyzed by the $2^{-\Delta \Delta \mathrm{CT}}$ method. All experiments were done in triplicate.
Protein extraction and western blot analysis. Total protein extracted in RIPA (Beyotime, China) buffer containing 1\% PMSF and phosphatase inhibitor from each subset, separated by SDS-PAGE and transferred to PVDF membranes. The membranes were blocked with skimmed milk and incubated (overnight, $4^{\circ} \mathrm{C}$ ) with an anti-NRP1 monoclonal antibody (1:1000, Abcam). After washings with TBS-T, membranes were incubated (room temperature, $1 \mathrm{~h}$ ) with horseradish peroxidase-conjugated secondary antibody and exposed to enhanced chemiluminescence reagent. To confirm equal protein loading, an anti- $\beta$-actin antibody (1:2000, Sigma, USA) was used to re-probe.

Cell culture and transfection. Huh7 and HCCLM3 cells purchased from the Chinese Academy of Sciences (Shanghai, China), all cells have STR profile report. Cells were grown in Dulbecco's modified Eagle's medium (DMEM, Gibco), 10\% $(\mathrm{v} / \mathrm{v})$ fetal bovine serum (Hyclone, USA). Cells were cultured in a humidified incubator (Thermo Fisher Scientific, USA) at $37^{\circ} \mathrm{C}$ and $5 \% \mathrm{CO}_{2}$. NRP1 small hairpin RNA (NRP1 shRNA) and negative control lentivirus (NRP1 shRNA NC) were produced by GeneChem (Shanghai, China) and transfected according to the manufacturer's instructions. Stably transfected cells were used for subsequent experiments.

Transwell assays, colony formation assays, and sphere formation assays. After trypsinization $(0.25 \%$ trypsin-EDTA, $\mathrm{BI})$, the cell concentration was adjusted to $2 \times 10^{5} \mathrm{cells} / \mathrm{ml}$. $600 \mu \mathrm{l}$ of complete medium containing $30 \%(\mathrm{v} / \mathrm{v})$ serum was added to the lower chamber and $200 \mu \mathrm{l}$ of cell suspension was added to the upper chamber, and cultured for $48 \mathrm{~h}$ for transwell assays.

Cells were seeded in 6-well plates (Corning, USA) for colony formation assays. After 2 weeks of culture, cells were fixed with $4 \%$ paraformaldehyde (Solarbio, China), stained with $0.5 \%$ crystal violet (Solarbio, China), observed and photographed. Image pro plus 6.0 was used to count the number of cells.

2000 cells/well were planted on ultra-low attachment surface 6-well plates (Corning, USA). After 8 days of culture, cells were counted and the images were taken (random 15 fields/well) under a stereomicroscope (Olympus, Japan). Image pro plus 6.0 was performed to measure the diameter of the spheres, diameter $>20 \mu \mathrm{m}$ was considered as formed spheres.

In vivo experiment. Four-week-old nude mice were purchased from Beijing Vitalstar Biotechnology Co, Ltd (Beijing, China) and maintained in strict pathogen-free conditions at the Animal Center housed by Gansu University of Traditional Chinese Medicine. The liver orthotopic transplantation model was produced, according to standard procedures previously described [15]. The mice were sacrificed after 6 weeks. The liver, lung, and tumor tissues were taken out, fixed with $4 \%$ formalin, and embedded in paraffin. All animals received appropriate care and all procedures followed guidelines established by the National Institutes of Health Guide for the Care and Use of Laboratory Animals. 
Flow cytometry and cell analysis. Phycoerythrin (PE)-conjugated CD133 antibody (cat. no. 130-110-962), PE-Vio770-conjugated CD13 antibody (cat. no. 130-120727), allophycocyanin (APC)-conjugated EpCAM antibody (cat. no. 130-111-000), APC-Vio770-conjugated CD44 antibody (cat. no. 130-113-339), and isotype control antibody (cat. no. 130-113-438, 130-113-440, 130-113-434, 130-113435 , respectively) were all purchased from Miltenyi Biotec (Bergisch Gladbach, Germany). The corresponding antibody was used according to the manufacturer's instructions. The percentage of CD133, CD13, EpCAM, and CD44 in the cell population was detected using BD LSRFortessa. All experiments were done in triplicate.

Bioinformatics analysis. Related genes of NRP1 were selected from the Cbioportal (https://www.cbioportal. org/), Coexpedia (http://www.coexpedia.org/), Ualcan (http://ualcan.path.uab.edu/), and Genecards (https:// www.genecards.org/) database, 123 intersection genes were screened out. FunRich 3.1.3 (http://www.funrich.org/) software was used for GO analysis and KEGG pathway analysis of NRP1 related genes.

Statistical analysis. Standard software (SPSS 24.0 for Windows; SPSS Inc., Chicago, IL, USA) was used for all statistical analyses and $\mathrm{p}<0.05$ was considered statistically significant. A Student's t-test or one-way ANOVA were used for comparisons of quantitative data of groups. Categorical data were subjected to chi-square testing. Overall survival (OS) and cumulative recurrence rates were estimated by the Kaplan-Meier method, and differences were determined using a log-rank test. Univariate and multivariate analyses were performed using Cox proportional hazards regression model.

\section{Results}

NRP1 drives the stem-like properties of HCC. To evaluate the regulation of NRP1 in liver cancer stem cells, small hairpin RNA (shRNA) was used to transfect HCCLM3 and Huh7 cells. To confirm that NRP1 shRNA suppressed the expression of NRP1, qRT-PCR and western blot were used to confirm the expression level of NRP1 mRNA and NRP1 protein. Our results showed that the expression of NRP1 mRNA and NRP1 protein decreased significantly after transfection of NRP1 shRNA (Figures 2A-2B). The cells expressing the surface markers (CD13/CD133/EpCAM/ CD44) of liver CSCs were detected by flow cytometry to investigate the effect of NRP1 on the CSCs population.

In HCCLM3 cells, the percentage of $\mathrm{CD}_{133}{ }^{+}, \mathrm{EpCAM}^{+}$, $\mathrm{CD}_{13}{ }^{+}, \mathrm{CD} 44^{+}$was significantly reduced in NRP1-sh1 transfected cells. The percentage of $\mathrm{CD}_{13}{ }^{+}$and $\mathrm{EpCAM}^{+}$was decreased by stably transfected NRP1-sh2 (Figure 1A).

The same experiment was performed in Huh7 cells to confirm the regulatory effect of NRP1 on liver CSCs. The decreased proportion of $\mathrm{CD}_{133}{ }^{+}, \mathrm{EpCAM}^{+}, \mathrm{CD}_{13}{ }^{+}, \mathrm{CD}_{4} 4^{+}$ was also observed after NRP1-sh1 transfection in Huh7 cells, the proportion of $\mathrm{CD}_{133^{+}}, \mathrm{CD} 13^{+}$was reduced by transfection with NRP1-sh2 (Figure 1B). In summary, these data indicate that in human HCC, NRP1 negatively regulates the populations of CSCs.

To investigate whether NRP1 knockdown affects the proliferation and self-renewal ability of HCC cells, we conducted the colony formation assay. In the colony formation analysis of HCCLM3 and Huh7 cell lines, a lack of proliferation and self-renewal was observed after NRP1 knockdown (Figures 1C, 1D). Sphere formation assays were performed to further validate the influence of NRP1 on the stem-like properties of HCC, the diameter of the spheres were significantly smaller when transfected with NRP1 shRNA compared with the negative control lentivirus transfection (Figures 1E, 1F). These results provide common evidence that NRP1 is essential for driving the properties of cells with stem cell characteristics in HCC.

Decreased NRP1 levels negatively correlated with metastasis of HCC cell lines. As the knockdown of NRP1 inhibits the expression of CSCs surface markers, we speculated that NRP1 is related to the migration and metastasis of HCC. First, we detected the expression level of NRP1 mRNA and NRP1 protein in human L02 hepatocytes and several liver cancer cell lines (HepG2, Huh7, HCCLM3) by qRT-PCR and western blot. The level of NRP1 mRNA and NRP1 protein in HCC cell lines was higher compared to L02 cells (Supplementary Figure S1A). To validate the effects of NRP1 on the migration capacity of HCC cells, HCCLM3 and Huh7 cells were transfected with NRP1 knockdown or negative control lentivirus. The transwell assays confirmed that the number of migrated cells of NRP1 shRNA transfected cells was significantly lower than negative control transfected cells (Figures 2C, 2D).

To investigate whether NRP1 knockdown also affects tumor formation and lung metastasis potential in vivo, HCCLM3 cells were transfected with NRP1 knockdown or negative control lentivirus, then the transfected cells were transplanted into the liver of nude mice in situ. After transplantation of the HCC cells into nude mice, all subsets succeeded in forming subcutaneous tumors (Supplementary Figure S1B). In the liver orthotopic transplantation model, pulmonary metastasis occurred in $100 \%(5 / 5)$ of the HCCLM3-NC grafted mice, which was higher than the rate observed in the HCCLM3-shNRP1 (1/5) grafts. The number of each grade of metastatic nodules stemming from the HCCLM3-NC grafts was also greater (Figure 2E). Thus, our findings suggested that high NRP1 expression could be related to the migration and metastasis of HCC.

NRP1 upregulation is indicative of poor prognosis and recurrence. We found that NRP1 promotes HCC metastasis is confirmed by in vivo and in vitro experiments. However, the clinical and prognostic characteristics of NRP1 in HCC patients still unknown. To address this issue, qRT-PCR analysis of tissue from 81 HCC patients (cohort $1, n=81$ ) revealed elevated expression of NRP1 in $64 \%$ of the tumor 

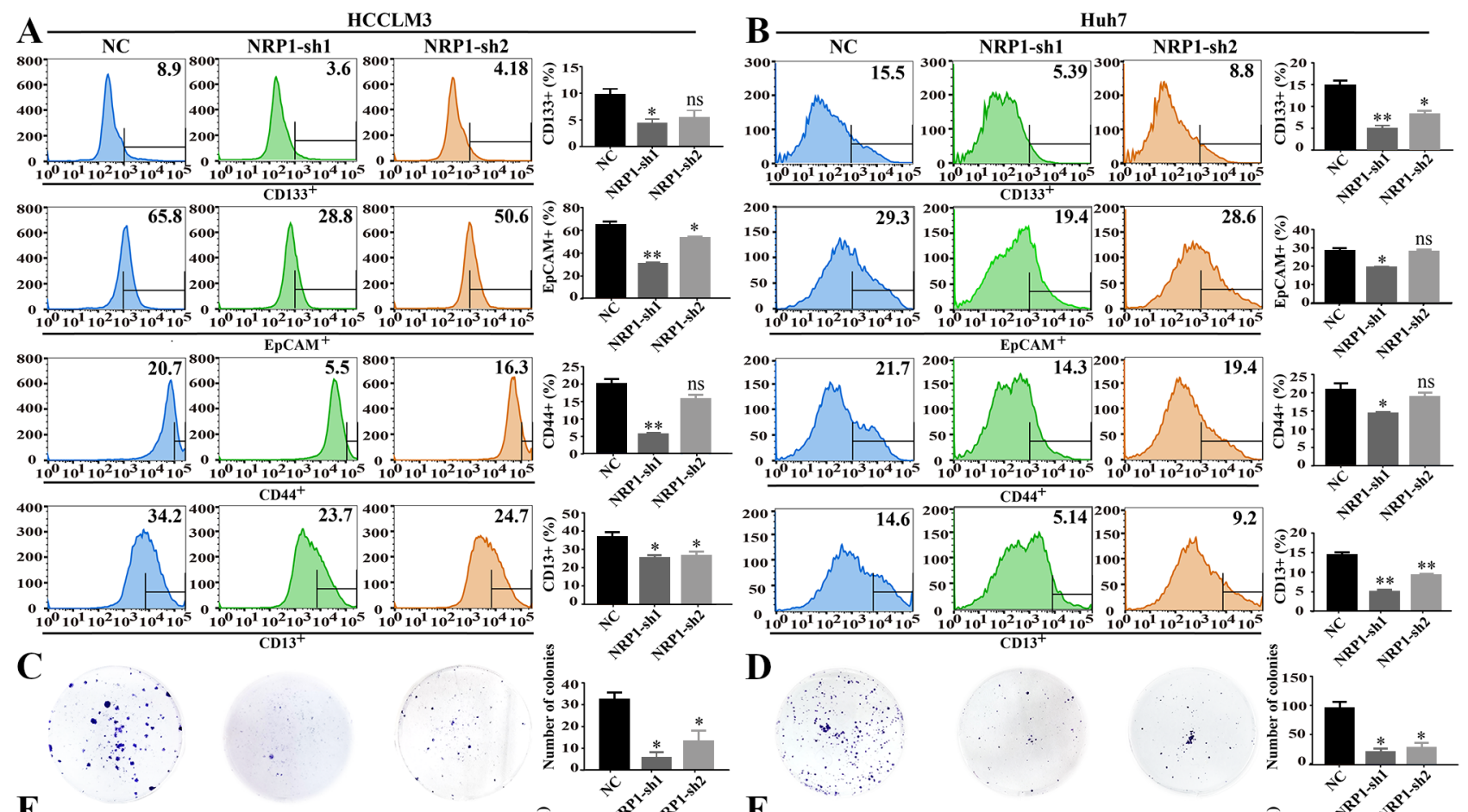

$\mathbf{E}$

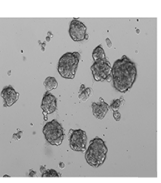

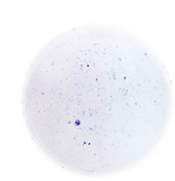

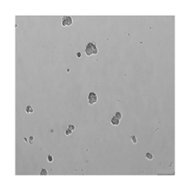

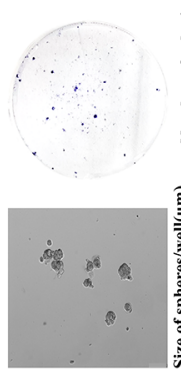
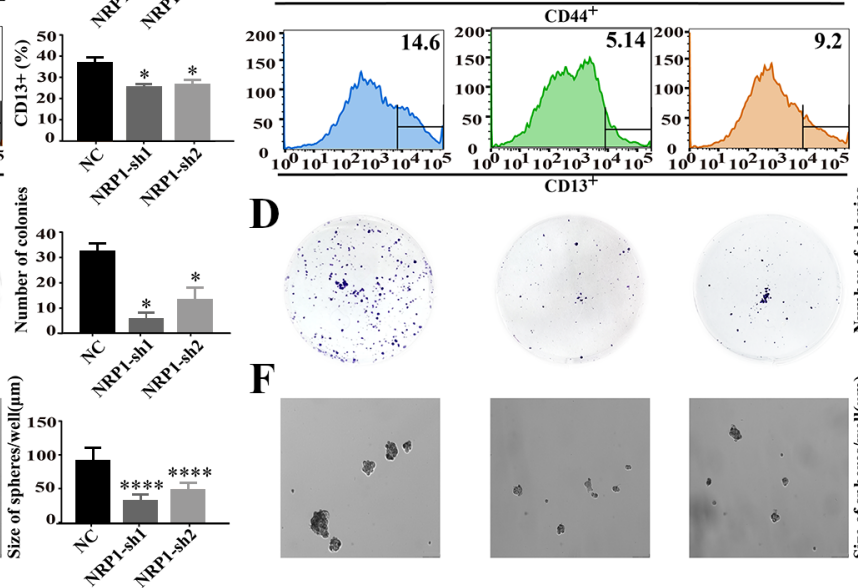

D

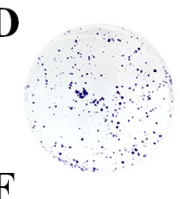

$\mathbf{F}$

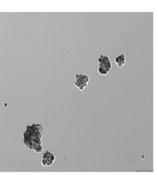

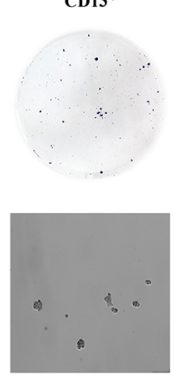

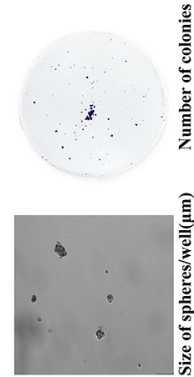

Figure 1. NRP1 drives the stem-like properties of HCC. A-B) NRP1 small hairpin RNA and negative control lentivirus transfected HCCLM3 and Huh7 cells for $72 \mathrm{~h}$. Flow cytometry results showing the percentage of CD133+, EpCAM+, CD44+, CD13+ in stably transfected cells. The ordinate represents the cell number, the abscissa indicates the fluorescence intensity. Three independent repeated tests showing with histogram. C-D). Colony formation of stably transfected HCCLM3 and Huh7 cells. Counting the number of cells with image-Pro plus 6.0. E-F) Image shows the sphere formation stably transfected HCCLM3 and Huh7 cells, histogram shows the diameter of the spheres in each well. One-way ANOVA was used to show a statistical difference. Results are shown as means $\pm \mathrm{SD} .{ }^{\star} \mathrm{p}<0.05,{ }^{* *} \mathrm{p}<0.01,{ }^{* *} \mathrm{p}<0.001,{ }^{\star * * *} \mathrm{p}<0.0001$, ns means no significance.

tissue samples compared to $36 \%$ of paracancer tissue samples (Figure 3A). Western blot detected NRP1 expression in tumor tissues and paracancer tissue of 16 randomly selected HCC patients (cohort 2, $\mathrm{n}=16$ ), the increased expression of NRP1 can be detected in $62.5 \%(10 / 16)$ of tumor tissues compared to $37.5 \%(6 / 16)$ of paracancer tissue (Figure $3 \mathrm{~B}$ ). NRP1 expression was scored by immunohistochemical staining in HCC samples from 239 patients (cohort 3, $\mathrm{n}=239$ ). Semi-quantitative analysis of NRP1 expression was performed based on the density of cells staining as follows: 0) $<5 \%$; 1) 6-35\%; 2) 36-70\%; 3) $>70 \%$ (the scoring standard of NRP1 expression is shown in Supplementary Figure S1C). Specimens with scores of 0 and 1 are regarded as low expression of NRP1 $\left(\mathrm{NRP} 1_{\text {Low }}\right)$, while specimens with scores of 2 and 3 are classified as high expression of NRP1 (NRP1 $\left.1_{\text {High }}\right)$. The OS (overall survival) between the $\mathrm{NRP} 1_{\text {High }}$ group and the NRP1 $1_{\text {Low }}$ group were analyzed.

As of the date of the last follow-up (March 2009), 57.3\% $(137 / 239)$ of the patients had died. In this cohort, OS of the NRP $1_{\text {High }}$ group was significantly lower than that of the $\mathrm{NRP} 1_{\text {Low }}$ group $(\log \mathrm{Rank}=24.953 ; \mathrm{p}=0.000)$ (Figure $\left.3 \mathrm{C}\right)$.

36 HCC tumors sampled from randomly recruited patients (cohort 4, $\mathrm{n}=16$; cohort 5, $\mathrm{n}=20$ ), 18 suffered recurrences and the remaining were recurrence-free at 2 years. Western blot and qRT-PCR were used to detect the expression of NRP1 in HCC patients with and without recurrence. The results showed that the expression level of NRP1 in patients with recurrence was higher than that in patients without recurrence (Figures $3 \mathrm{D}, 3 \mathrm{E}$ ).

The expression of NRP1 is an independent prognostic factor affecting OS. We analyzed the clinical data of 239 HCC patients whose samples underwent immunohistochemical staining. Clinical parameters collected included gender, age, AFP levels, Child-Pugh scores, tumor sizes, tumor microvascular invasion, pathological stages, and so on.

Table 1 shows the correlation analysis results between the expression of NRP1 and clinicopathological characteristics. It can be seen that the expression of NRP1 is correlated with 
A

HCCLM3
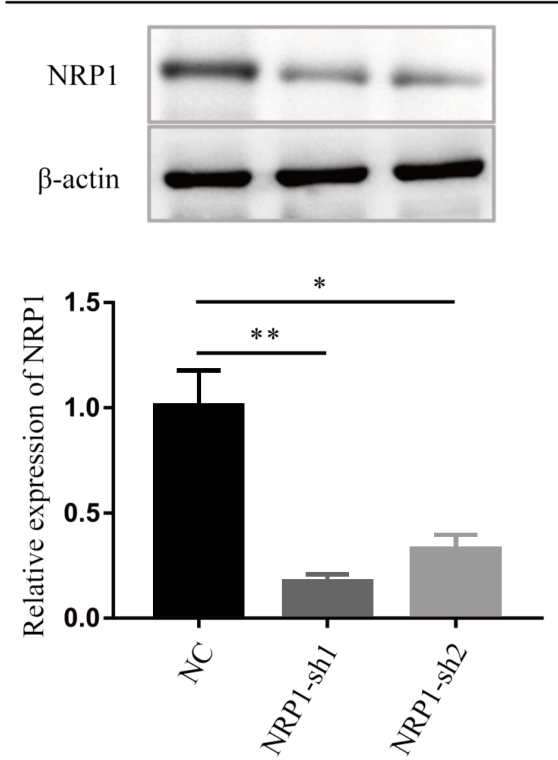

$\mathrm{C}$

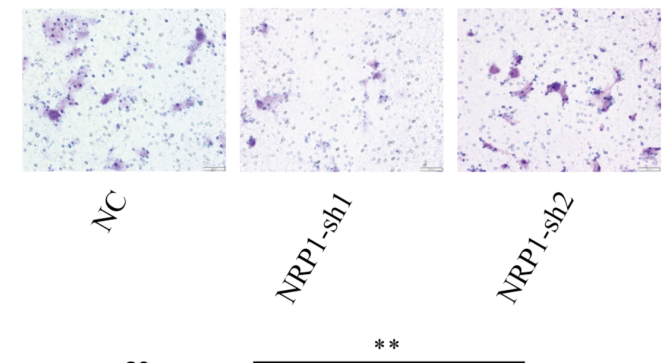

E

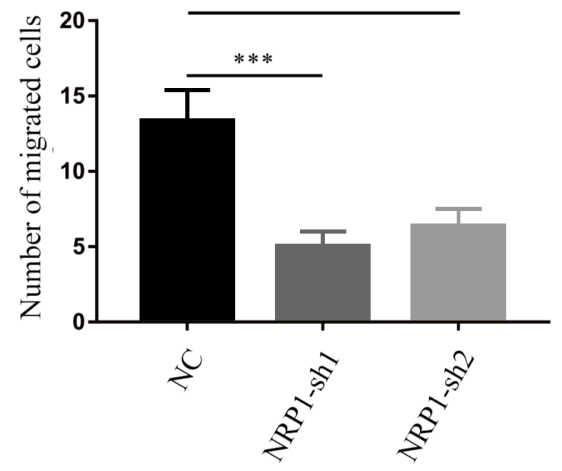

B
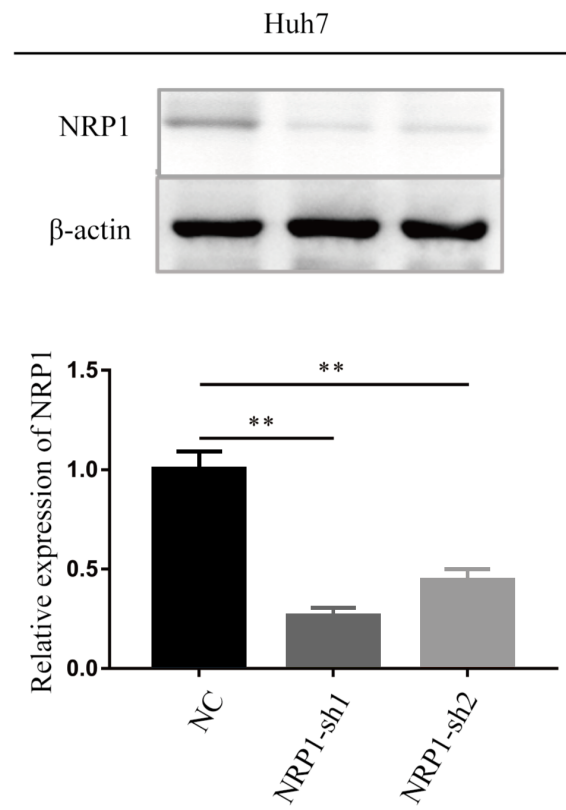

$\mathrm{D}$
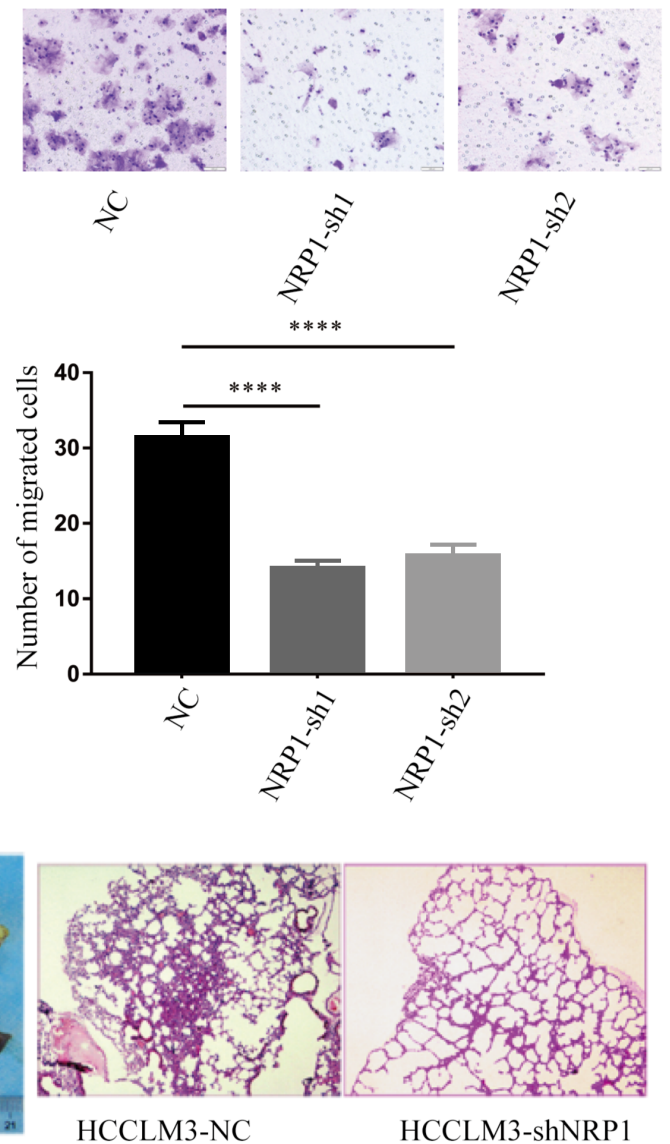

Figure 2. Decreased NRP1 levels negatively correlated with metastasis of HCC cell lines. A-B) Western blot and real-time quantitative PCR were used to detect the expression of NRP1 mRNA and NRP1 protein in HCCLM3 and Huh7 cells to verify the knockdown effect. C-D) The effect of NRP1 knockdown on the migration of HCCLM3 and Huh7 cells was evaluated by transwell assays. E) Liver orthotopic transplantation model was produced through transfection of HCCLM3-NC and HCCLM3-shNRP1 cells. Lung metastatic nodules were observed. One-way ANOVA was used to show a statistical difference. Results are shown as means $\pm S D .{ }^{\star} p<0.05,{ }^{\star *} p<0.01,{ }^{\star * *} p<0.001,{ }^{\star * * *} p<0.0001$. 
A

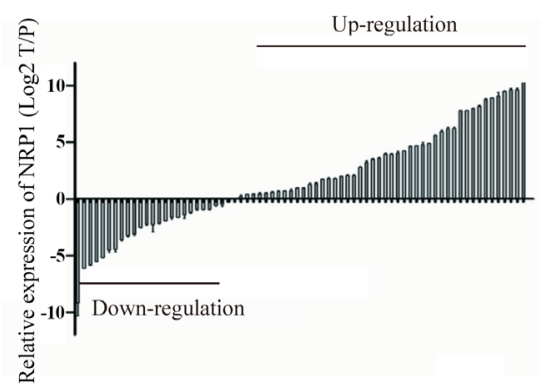

C

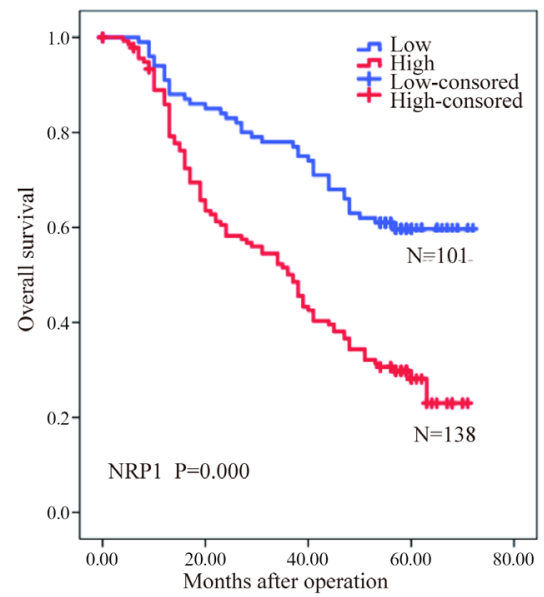

B

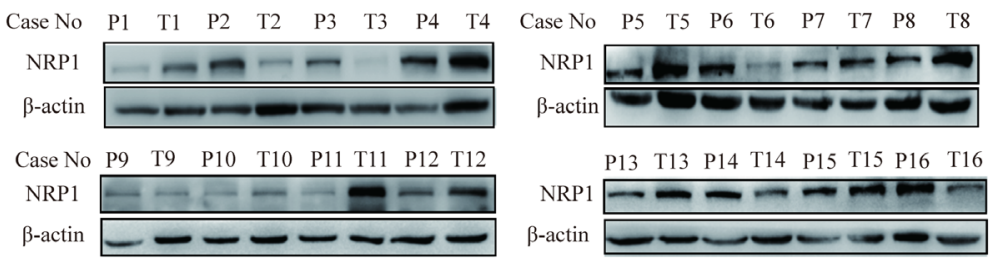

D

E

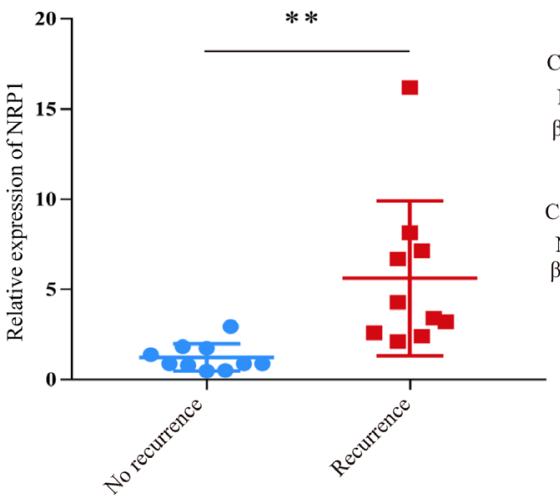

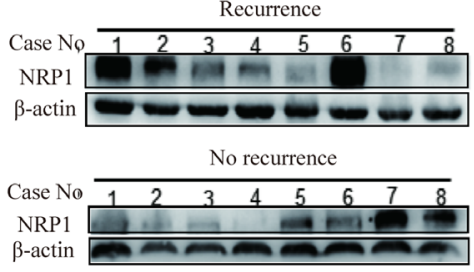

Figure 3. NRP1 upregulation is indicative of poor prognosis and recurrence. A) The expression of NRP1 was detected by $\mathrm{qRT}$-PCR in 81 cases of the tumor and adjacent tissues (cohort1). B) Western blot showed the expression of NRP1 in 16 cases of the tumor and adjacent tissues (cohort 2). C) Kaplan-Meier analysis between OS and 239 HCC patients with high or low expression of NRP1 (cohort 3 ). D) The expression of NRP1 was detected by $\mathrm{qRT}$-PCR in 10 recurrence and 10 non-recurrence HCC patients (cohort 4 ), $t$-test, ${ }^{* *}$ p $<0.01$. E) Western blot showed the expression of NRP1 in 8 recurrence and 8 non-recurrence patients (cohort 5 ). T means tumor tissue, $P$ means paracancer tissue.

the presence or absence of vascular invasion $(\mathrm{p}=0.007)$, but has no correlation with other clinical indicators.

Using COX regression analysis, the univariate analysis showed that the risk factors affecting liver cancer OS were portal vein tumor thrombus (1.590 (1.007-2.510), $\mathrm{p}=0.047)$ and the expression of NRP1 (2.465 (1.703-3.570), $\mathrm{p}=0.000)$ (Table 2).

Multivariate analysis of the single-factor risk factors showed that the expression of NRP1 (2.292 (1.578-3.329), $\mathrm{p}=0.000)$ is an independent prognostic factor that affects OS (Table 2).

NRP1 functions by regulating the EMT signaling pathway in vitro. To further evaluate the mechanism of NRP1 in promoting HCC metastasis, recurrence, and poor prognosis bioinformatics methods were used to screen out 123 NRP1 related genes (Supplementary Table S1) from four databases (Figure 4A). KEGG enrichment analysis of related genes revealed that NRP1 mainly functions through the EMT signaling pathway (Figure 4B). Western blot analysis showed that knockdown of NRP1 restored E-cadherin expression and reduced $\mathrm{N}$-cadherin and Vimentin expres- sion in HCCLM3 and Huh7 cells (Figure 4C). Verified that NRP1 promotes HCC cells' metastasis by regulating the EMT signaling pathway.

\section{Discussion}

A lot of convincing evidence has revealed that the existence of cancer stem cells (CSCs) is responsible for HCC metastasis, recurrence, and heterogeneity [16]. Here, we identified that the knockdown of NRP1 suppresses the population of CSCs representative surface markers $\left(\mathrm{CD}_{133}{ }^{+} / \mathrm{EpCAM}^{+} /\right.$ $\mathrm{CD}_{13}{ }^{+}$), inhibits the migration of HCC cells, and the recurrence and prognosis of HCC patients.

It has been recently demonstrated that NRP1 has tumorinducing properties [17], promotes survival of CSCs [18], enhances resistance to chemotherapy drugs [11], the VEGF-Nrp1 axis regulates tumor initiation and stemness [19], and promotes the viability of glioma stem-like cells and tumor growth [20], NRP1 may be a useful biomarker for tumor-initiating cells or CSCs. Our research focuses on the effect of NRP1 on liver CSCs and shows the expression of 
Table 1. Correlation between the factors and clinicopathological characteristics in HCC $(n=239)$.

\begin{tabular}{|c|c|c|c|}
\hline \multirow{2}{*}{$\begin{array}{l}\text { Clinicopathological } \\
\text { indexes }\end{array}$} & \multicolumn{3}{|c|}{ NRP1 } \\
\hline & Low & High & p-value \\
\hline \multicolumn{4}{|l|}{ HBV } \\
\hline Negative & $68(63.7 \%)$ & $96(69.6 \%)$ & \multirow{2}{*}{0.713} \\
\hline Positive & $33(32.7 \%)$ & $42(30.4 \%)$ & \\
\hline \multicolumn{4}{|c|}{ Portal vein tumor thrombus } \\
\hline No & $90(89.1 \%)$ & $117(84.8 \%)$ & \multirow{2}{*}{0.332} \\
\hline Yes & $11(10.9 \%)$ & $21(15.2 \%)$ & \\
\hline \multicolumn{4}{|c|}{ Extrahepatic bile duct/gallbladder invasion } \\
\hline No & $95(94.1 \%)$ & $133(96.4 \%)$ & \multirow{2}{*}{0.398} \\
\hline Yes & $6(5.9 \%)$ & $5(3.6 \%)$ & \\
\hline \multicolumn{4}{|l|}{ Gender } \\
\hline Male & $91(90.1 \%)$ & $124(89.9 \%)$ & \multirow{2}{*}{0.951} \\
\hline Female & $10(9.9 \%)$ & $14(10.1 \%)$ & \\
\hline \multicolumn{4}{|l|}{ Age (year) } \\
\hline$\leq 50$ & $31(30.7 \%)$ & $59(42.8 \%)$ & \multirow{2}{*}{0.057} \\
\hline$>50$ & $70(69.3 \%)$ & $79(57.2 \%)$ & \\
\hline \multicolumn{4}{|l|}{ Vascular invasion } \\
\hline No & $61(60.4 \%)$ & $59(42.8 \%)$ & \multirow{2}{*}{0.007} \\
\hline Yes & $40(39.6 \%)$ & $79(57.2 \%)$ & \\
\hline \multicolumn{4}{|l|}{ Tumor number } \\
\hline Single & $92(91.1 \%)$ & $122(88.4 \%)$ & \multirow[b]{2}{*}{0.503} \\
\hline Multiple & $9(8.9 \%)$ & $16(11.6 \%)$ & \\
\hline \multicolumn{4}{|l|}{ Metastasis } \\
\hline No & $95(94.1 \%)$ & $127(92.0 \%)$ & \multirow{2}{*}{0.546} \\
\hline Yes & $6(5.9 \%)$ & $11(8.0 \%)$ & \\
\hline \multicolumn{4}{|l|}{ Liver cirrhosis } \\
\hline No & $61(60.4 \%)$ & $89(64.5 \%)$ & \multirow{2}{*}{0.518} \\
\hline Yes & $40(39.6 \%)$ & $49(35.5 \%)$ & \\
\hline \multicolumn{4}{|l|}{ Tumor size $(\mathrm{cm})$} \\
\hline$\leq 5$ & $45(44.6 \%)$ & $50(36.2 \%)$ & \multirow{2}{*}{0.194} \\
\hline$>5$ & $56(55.4 \%)$ & $88(63.8 \%)$ & \\
\hline \multicolumn{4}{|l|}{ Pathological stages } \\
\hline I/II & $18(17.8 \%)$ & $20(14.5 \%)$ & \multirow{2}{*}{0.487} \\
\hline III/IV & $83(82.2 \%)$ & $118(85.5 \%)$ & \\
\hline \multicolumn{4}{|l|}{ AFP (ng/ml) } \\
\hline$\leq 20$ & $16(15.8 \%)$ & $28(20.3 \%)$ & \multirow{2}{*}{0.381} \\
\hline$>20$ & $85(84.2 \%)$ & $110(79.7 \%)$ & \\
\hline
\end{tabular}

CSCs surface markers and self-renewal observed through colony formation are decreased after NRP1 knockdown. CD133 and EpCAM are the most important markers of liver CSCs [21]. Our results showed that after knocking down NRP1, the expression of CD133 and EPCAM decreased significantly, thus proving the important role of NRP1 in liver CSCs, which consistent with the research of Liu et al. [22]. They proposed that miR-148b plays key role in maintaining the CSCs characteristics of side population cells by targeting NRP1. OCT4, SOX2, and NANOG constitute the core transcription factors that regulate stem cell self-renewal, and are commonly used to characterize CSCs, but they are mainly distributed in
Table 2. Univariate and multivariate analyses of prognostic factors in HCC $(n=239)$.

\begin{tabular}{|c|c|c|}
\hline \multirow{2}{*}{ Variable } & \multicolumn{2}{|l|}{ OS } \\
\hline & HR (95\% CI) & p-value \\
\hline \multicolumn{3}{|l|}{ Univariate analysis } \\
\hline HBV & $1.280(0.901-1.819)$ & 0.168 \\
\hline Portal vein tumor thrombus & $1.590(1.007-2.510)$ & 0.047 \\
\hline Extrahepatic bile duct/gallbladder invasion & $1.597(0.746-3.421)$ & 0.228 \\
\hline Gender (female vs. male) & $0.726(0.401-1.313)$ & 0.289 \\
\hline Age, year ( $\leq 50$ vs. $>50)$ & $1.003(0.717-1.402)$ & 0.988 \\
\hline Vascular invasion & $1.219(0.871-1.701)$ & 0.249 \\
\hline Tumor number (single vs. multiple) & $1.610(0.979-2.646)$ & 0.060 \\
\hline Metastasis & $1.259(0.696-2.278)$ & 0.446 \\
\hline Liver cirrhosis & $0.946(0.667-1.342)$ & 0.755 \\
\hline Tumor size, $\mathrm{cm}(\leq 5$ vs. $>5)$ & $1.089(0.774-1.533)$ & 0.623 \\
\hline Pathological stages (I/II vs. III/ IV) & $1.030(0.652-1.626)$ & 0.900 \\
\hline AFP, ng/ml ( $\leq 20$ vs. $>20)$ & $1.254(0.801-1.963)$ & 0.322 \\
\hline NRP1 (low vs. high) & $2.465(1.703-3.570)$ & $<0.001$ \\
\hline \multicolumn{3}{|l|}{ Multivariate analysis } \\
\hline Portal vein tumor thrombus & $1.517(0.957-2.403)$ & 0.076 \\
\hline NRP1 (low vs. high) & $2.292(1.578-3.329)$ & $<0.001$ \\
\hline
\end{tabular}

A

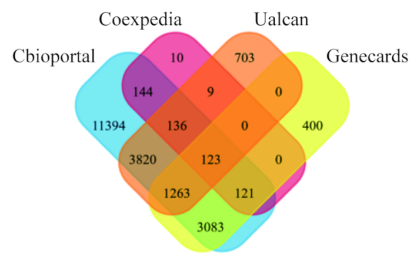

B Biological pathway
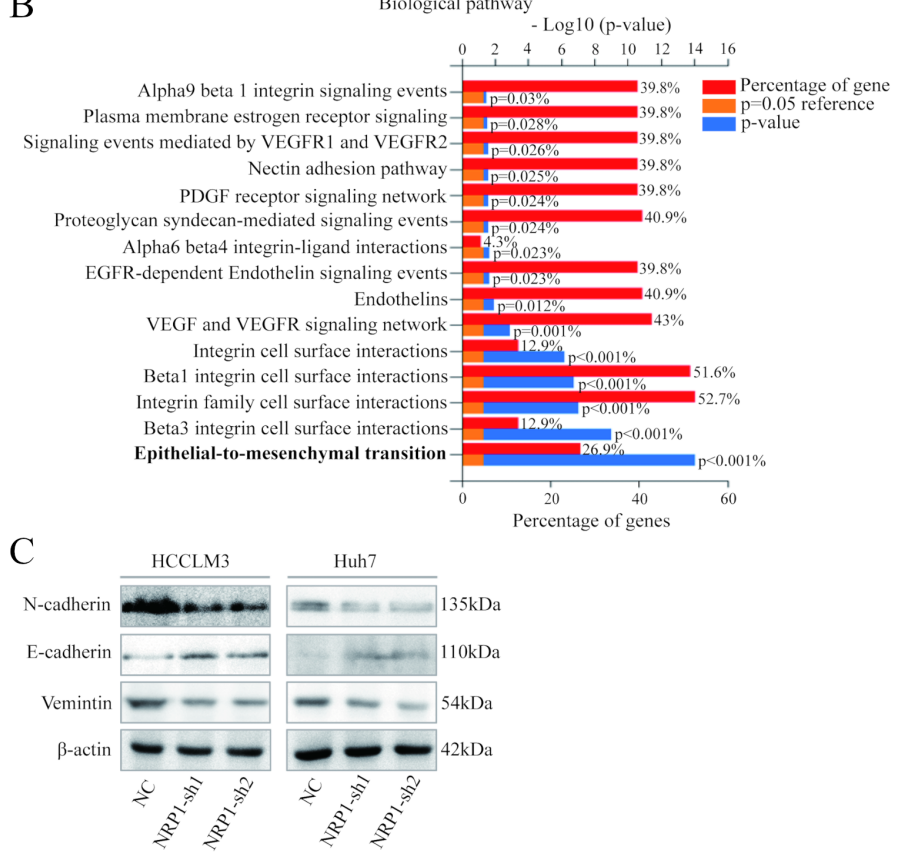

Figure 4. NRP1 functions by regulating the EMT signaling pathway in vitro. A) Related genes of NRP1 were chosen in hepatocellular carcinoma (HCC) from the Cbioportal, Coexpedia, Ualcan, and Genecards, 123 intersection genes were screened out. B) KEGG enrichment analysis of NRP1 related genes by Funrich. C) Expression of E-cadherin, N-cadherin, and Vimentin in HCCLM3 and Huh7 cells with NRP1 knockdown and negative control were detected by western blot. $\beta$-Actin was used as a loading control. 
the nucleus and are not easily detected [23]. The development of membrane surface markers such as NRP1 for CSCs characterization is crucial. NRP1 may be used as a new CSCs surface marker, which is a very interesting aspect worth studying.

Knockdown or overexpression of different stemness-related genes of CSCs to construct super-stem maintenance CSCs (super-CSCs). After establishing this super-CSCs model, we will investigate the characteristics of CSCs more deeply and effectively. Our laboratory has made some efforts in this aspect. NRP1 is one of the preliminary attempts to construct a super-CSCs model. The identification and sorting of new CSCs markers based on the super-CSCs model may be a promising prospect and direction for future CSCs researches.

Accumulating evidence shows that NRP1 is a tumorpromoting factor, upregulated in a variety of tumors, promoting proliferation and metastasis [24]. From the perspective of NRP1 promoting the proportion of surface markers of liver CSCs, our research inferred that NRP1 is related to the clinical features of HCC. Through qRT-PCR, western blot, and immunohistochemistry we observed that NRP1 is highly expressed in HCC and an elevated level of NRP1 promotes HCC recurrence and poor prognosis. The study of Lin et al. [25] showed that serum NRP1 has better diagnostic performance for HCC than AFP, and knocking down NRP1 inhibits the phenotype of HCC cells. Lin's and our research together suggest that NRP1 can be used as a diagnostic and potential prognostic target for HCC.

In summary, our study provides a better understanding of the molecular mechanisms of NRP1 involved in HCC metastasis. Furthermore, our results suggest a potential role of NRP1 as a biomarker of disease progression and prognosis in HCC patients.

Supplementary information is available in the online version of the paper.

Acknowledgments: This work was supported by the Science and Technology Major Project of Gansu province (1602FKDA001), the Talent innovation and Entrepreneurship Program of Lanzhou City (2016-RC-57), Gansu Province Science and Technology Plan Project Mission Statement (18JR2TA018), the Science and Technology Project of Chengguan District (2017SHFZ0014) and Gansu Province Health Industry Scientific Research Project (GSWSKY-2015-490).

\section{References}

[1] SINGAI A, LAMPERTICO P, NAHON P. Epidemiology and surveillance for hepatocellular carcinoma: New trends. J Hepatol 2020; 72: 250-261. https://doi.org/10.1016/j. jhep.2019.08.025

[2] DATTILO R, MOTTINI C, CAMERA E, LAMOLINARA A, AUSLANDER $\mathrm{N}$ et al. Pyrvinium pamoate induces death of triple-negative breast cancer stem-like cells and reduces metastases through effects on lipid anabolism. Cancer Res 2020. https://doi.org/10.1158/0008-5472.CAN-19-1184
[3] GARCIA-MAYEA Y, MIR C, MASSON F, PACIUCCI R, ME LL. Insights into new mechanisms and models of cancer stem cell multidrug resistance. Semin Cancer Biol 2020; 60: 166-180. https://doi.org/10.1016/j.semcancer.2019.07.022

[4] WU J, ZHU P, LU T, DU Y, WANG Y et al. The long noncoding RNA LncHDAC2 drives the self-renewal of liver cancer stem cells via activation of Hedgehog signaling. J Hepatol 2019; 70: 918-929. https://doi.org/10.1016/j. jhep.2018.12.015

[5] COLE AJ, FAYOMI AP, ANYAECHE VI, BAI S, BUCKANOVICH RJ. An evolving paradigm of cancer stem cell hierarchies: therapeutic implications. Theranostics 2020; 10: 3083-3098. https://doi.org/10.7150/thno.41647

[6] WEI Y, GUO S, TANG J, WEN J, WANG H et al. MicroRNA-19b-3p suppresses gastric cancer development by negatively regulating neuropilin-1. Cancer Cell Int 2020; 20: 193. https://doi.org/10.1186/s12935-020-01257-0

[7] KO JH, KWON HS, KIM B, MIN G, SHIN C et al. Preclinical Efficacy and Safety of an Anti-Human VEGFA and Anti-Human NRP1 Dual-Targeting Bispecific Antibody (IDB0076). Biomolecules 2020; 10: 919. https://doi.org/10.3390/ biom 10060919

[8] MEI B, CHEN J, YANG N, PENG Y. The regulatory mechanism and biological significance of the Snail-miR590-VEGFR-NRP1 axis in the angiogenesis, growth and metastasis of gastric cancer. Cell Death Dis 2020; 11: 241. https://doi. org/10.1038/s41419-020-2428-x

[9] RIZZOLIO S, CAGNONI G, BATTISTINI C, BONELLI S, ISELLA $C$ et al. Neuropilin-1 upregulation elicits adaptive resistance to oncogene-targeted therapies. J Clin Invest 2018; 128: 3976-3990. https://doi.org/10.1172/JCI99257

[10] MA LL, GUO LL, LUO Y, LIU GL, LEI Y et al. Cdc42 subcellular relocation in response to VEGF/NRP1 engagement is associated with the poor prognosis of colorectal cancer. Cell Death Dis 2020; 11: 171. https://doi.org/10.1038/s41419020-2370-y

[11] CHU WM, SONG XM, YANG XM, MA L, ZHU J et al. Neuropilin-1 promotes epithelial-to-mesenchymal transition by stimulating nuclear factor-kappa B and is associated with poor prognosis in human oral squamous cell carcinoma. PLoS One 2014; 9: e101931. https://doi.org/10.1371/journal. pone. 0101931

[12] DING Z, DU W, LEI Z, ZHANG Y, ZHU J et al. Neuropilin 1 modulates TGFbetalinduced epithelialmesenchymal transition in nonsmall cell lung cancer. Int J Oncol 2020; 56: 531-543. https://doi.org/10.3892/ijo.2019.4938

[13] LIU C, SOMASUNDARAM A, MANNE S, GOCHER AM, SZYMCZAK-WORKMAN AL et al. Neuropilin-1 is a T cell memory checkpoint limiting long-term antitumor immunity. Nat Immunol 2020. https://doi.org/10.1038/s41590-0200733-2

[14] OVERACRE-DELGOFFE AE, CHIKINA M, DADEY $\mathrm{RE}$, YANO $\mathrm{H}$, BRUNAZZI EA et al. Interferon-gamma Drives Treg Fragility to Promote Anti-tumor Immunity. Cell 2017; 169: 1130-1141 e1111. https://doi.org/10.1016/j. cell.2017.05.005 
[15] LI CX, LING CC, SHAO Y, XU A, LI XC et al. CXCL10/ CXCR3 signaling mobilized-regulatory $\mathrm{T}$ cells promote liver tumor recurrence after transplantation. J Hepatol 2016; 65: 944-952. https://doi.org/10.1016/j.jhep.2016.05.032

[16] HUANG T, SONG X, XU D, TIEK D, GOENKA A et al. Stem cell programs in cancer initiation, progression, and therapy resistance. Theranostics 2020; 10: 8721-8743. https://doi. org/10.7150/thno.41648

[17] JIMENEZ-HERNANDEZ LE, VAZQUEZ-SANTILLAN K, CASTRO- OROPEZA R, MARTINEZ-RUIZ G, MUNOZ-

GALINDO L et al. NRP1-positive lung cancer cells possess tumor-initiating properties. Oncol Rep 2018; 39: 349-357. https://doi.org/10.3892/or.2017.6089

[18] GRUN D, ADHIKARY G, ECKERT RL. NRP-1 interacts with GIPC1 and SYX to activate p38 MAPK signaling and cancer stem cell survival. Molecular carcinogenesis 2019; 58: 488-499. https://doi.org/10.1002/mc. 22943

[19] BECK B, DRIESSENS G, GOOSSENS S, YOUSSEF KK, KUCHNIO A et al. A vascular niche and a VEGF-Nrp1 loop regulate the initiation and stemness of skin tumours. Nature 2011; 478: 399-403. https://doi.org/10.1038/nature 10525
[20] HAMERLIK P, LATHIA JD, RASMUSSEN R, WU Q, BARTKOVA J et al. Autocrine VEGF-VEGFR2-Neuropilin-1 signaling promotes glioma stem-like cell viability and tumor growth. The Journal of experimental medicine 2012; 209: 507-520. https://doi.org/10.1084/jem.20111424

[21] CASTELli G, PELOSI E, TESTA U. Liver Cancer: Molecular Characterization, Clonal Evolution and Cancer Stem Cells. Cancers (Basel) 2017; 9. https://doi.org/10.3390/cancers9090127

[22] LIU Q, XU Y, WEI S, GAO W, CHEN L et al. miRNA-148b suppresses hepatic cancer stem cell by targeting neuropilin-1. Bioscience reports 2015; 35. https://doi.org/10.1042/ bsr20150084

[23] KAUFHOLD S, GARBAN H, BONAVIDA B. Yin Yang 1 is associated with cancer stem cell transcription factors (SOX2, OCT4, BMI1) and clinical implication. J Exp Clin Cancer Res 2016; 35: 84. https://doi.org/10.1186/s13046-016-0359-2

[24] DUMOND A, PAGES G. Neuropilins, as Relevant Oncology Target: Their Role in the Tumoral Microenvironment. Front Cell Dev Biol 2020; 8: 662. https://doi.org/10.3389/ fcell.2020.00662

[25] LIN J, ZHANG Y, WU J, LI L, CHEN N et al. Neuropilin 1 (NRP1) is a novel tumor marker in hepatocellular carcinoma. Clin Chim Acta 2018; 485: 158-165. https://doi. org/10.1016/j.cca.2018.06.046 
https://doi.org/10.4149/neo_2020_200914N982

\section{Loss of neuropilin1 inhibits liver cancer stem cells population and blocks metastasis in hepatocellular carcinoma via epithelial-mesenchymal transition}

Xun $\mathrm{LI}^{1,2, *}$, Yongqiang $\mathrm{ZHOU}^{3}$, JinJing $\mathrm{HU}^{2,4}$, Zhongtian BAI ${ }^{1}$, Wenbo MENG ${ }^{1}$, Lei ZHANG ${ }^{1}$, Xiaojing SONG ${ }^{1}$, Yongjian $\mathrm{WEI}^{3}$, Jun YAN ${ }^{1}$, Yihua ZHOU ${ }^{3}$

\section{Supplementary Information}

Supplementary Table S1. Information on NRP1 related genes.

\begin{tabular}{|c|c|c|}
\hline Genes & Genes description & $\begin{array}{l}\text { Biological } \\
\text { functions in } \\
\text { HCC }\end{array}$ \\
\hline NRP1 & Neuropilin 1 & Prognosis \\
\hline CD93 & CD93 molecule & Prognosis \\
\hline DAB2 & DAB adaptor protein 2 & Metastasis \\
\hline TGFBR2 & Transforming growth factor beta receptor 2 & Invasion \\
\hline COL4A1 & Collagen type IV alpha 1 chain & Metastasis \\
\hline VIM & Vimentin & Metastasis \\
\hline LAMB1 & laminin subunit beta 1 & No results \\
\hline ZEB1 & zinc finger E-box binding homeobox 1 & Metastasis \\
\hline NID2 & nidogen 2 & Prognosis \\
\hline LAMA4 & laminin subunit alpha 4 & Metastasis \\
\hline COL6A2 & collagen type VI alpha 2 chain & No results \\
\hline VCAN & Versican & Metastasis \\
\hline LAMC1 & laminin subunit gamma 1 & Prognosis \\
\hline COL4A2 & collagen type IV alpha 2 chain & Prognosis \\
\hline PDGFRA & platelet derived growth factor receptor alpha & No results \\
\hline CAV1 & caveolin 1 & Metastasis \\
\hline AXL & AXL receptor tyrosine kinase & Invasion \\
\hline PDGFRB & platelet derived growth factor receptor beta & No results \\
\hline COL6A3 & collagen type VI alpha 3 chain & No results \\
\hline TCF4 & transcription factor 4 & Migration \\
\hline KCTD12 & $\begin{array}{l}\text { potassium channel tetramerization domain } \\
\text { containing } 12\end{array}$ & No results \\
\hline ANXA1 & annexin $\mathrm{A} 1$ & Prognosis \\
\hline CD163 & CD163 molecule & No results \\
\hline FSTL1 & follistatin like 1 & No results \\
\hline IGFBP7 & insulin like growth factor binding protein 7 & No results \\
\hline COL3A1 & collagen type III alpha 1 chain & No results \\
\hline MMRN2 & multimerin 2 & No results \\
\hline SPARC & secreted protein acidic and cysteine rich & Tumor growth \\
\hline MSN & Moesin & Metastasis \\
\hline COL5A1 & collagen type $\mathrm{V}$ alpha 1 chain & No results \\
\hline HSPG2 & heparan sulfate proteoglycan 2 & No results \\
\hline FERMT2 & fermitin family member 2 & Metastasis \\
\hline GJA1 & gap junction protein alpha 1 & No results \\
\hline CD44 & CD44 molecule & Metastasis \\
\hline PRKD3 & protein kinase D3 & No results \\
\hline CDH5 & cadherin 5 & No results \\
\hline ETS1 & ETS proto-oncogene 1, transcription factor & Progression \\
\hline PRKCH & protein kinase $\mathrm{C}$ eta & No results \\
\hline TIMP2 & TIMP metallopeptidase inhibitor 2 & No results \\
\hline ARHGAP29 & Rho GTPase activating protein 29 & No results \\
\hline HEPH & Hephaestin & No results \\
\hline MEF2C & myocyte enhancer factor $2 \mathrm{C}$ & Malignancy \\
\hline
\end{tabular}

\begin{tabular}{|c|c|c|}
\hline Genes & Genes description & $\begin{array}{l}\text { Biological } \\
\text { functions in } \\
\text { HCC }\end{array}$ \\
\hline CXCL12 & C-X-C motif chemokine ligand 12 & Metastasis \\
\hline DPYSL2 & dihydropyrimidinase like 2 & No results \\
\hline PMP22 & peripheral myelin protein 22 & No results \\
\hline WWTR1 & WW domain containing transcription regulator 1 & Prognosis \\
\hline COL1A1 & collagen type I alpha 1 chain & Invasion \\
\hline GNB4 & G protein subunit beta 4 & No results \\
\hline PLXND1 & plexin D & No results \\
\hline CALCRL & calcitonin receptor like receptor & No results \\
\hline GNG12 & G protein subunit gamma 12 & No results \\
\hline CRIM1 & cysteine rich transmembrane BMP regulator 1 & No results \\
\hline RGS5 & regulator of $\mathrm{G}$ protein signaling 5 & Metastasis \\
\hline ATP2C1 & ATPase secretory pathway $\mathrm{Ca} 2+$ transporting 1 & No results \\
\hline TCF7L2 & transcription factor 7 like 2 & Progression \\
\hline EDNRA & endothelin receptor type A & No results \\
\hline SH2B3 & SH2B adaptor protein 3 & No results \\
\hline FCGR2A & Fc fragment of IgG receptor IIa & No results \\
\hline LCP2 & lymphocyte cytosolic protein 2 & No results \\
\hline SPP1 & secreted phosphoprotein 1 & Progression \\
\hline ITGA6 & integrin subunit alpha 6 & No results \\
\hline TUBA1A & tubulin alpha 1a & No results \\
\hline FPR3 & formyl peptide receptor 3 & No results \\
\hline $\mathrm{NOTCH} 2$ & notch receptor 2 & Invasion \\
\hline RBBP7 & $\begin{array}{l}\text { RB binding protein } 7 \text {, chromatin remodeling } \\
\text { factor }\end{array}$ & No results \\
\hline PTGER4 & prostaglandin E receptor 4 & No results \\
\hline FBN1 & fibrillin 1 & No results \\
\hline PTPRB & protein tyrosine phosphatase receptor type $B$ & Migration \\
\hline LPAR6 & lysophosphatidic acid receptor 6 & No results \\
\hline PLD1 & phospholipase D1 & Migration \\
\hline NCK2 & NCK adaptor protein 2 & No results \\
\hline SNRK & SNF related kinase & No results \\
\hline TUBB6 & tubulin beta 6 class $\mathrm{V}$ & No results \\
\hline TNS3 & tensin 3 & No results \\
\hline СТВP2 & C-terminal binding protein 2 & Invasion \\
\hline PTPRM & protein tyrosine phosphatase receptor type $\mathrm{M}$ & No results \\
\hline PKP4 & plakophilin 4 & No results \\
\hline COL5A2 & collagen type $V$ alpha 2 chain & No results \\
\hline ITGA3 & integrin subunit alpha 3 & Proliferation \\
\hline ST8SIA4 & $\begin{array}{l}\text { ST8 alpha-N-acetyl-neuraminide alpha-2,8- } \\
\text { sialyltransferase } 4\end{array}$ & No results \\
\hline SPRY1 & sprouty RTK signaling antagonist 1 & Prognosis \\
\hline GSN & Gelsolin & Prognosis \\
\hline NRIP1 & nuclear receptor interacting protein 1 & No results \\
\hline
\end{tabular}


Supplementary Table S1. Continued ...

\begin{tabular}{|c|c|c|c|c|c|}
\hline Genes & Genes description & $\begin{array}{l}\text { Biological } \\
\text { functions in } \\
\text { HCC }\end{array}$ & Genes & Genes description & $\begin{array}{l}\text { Biological } \\
\text { functions in } \\
\text { HCC }\end{array}$ \\
\hline \multirow[t]{2}{*}{ LTBP1 } & \multirow{2}{*}{$\begin{array}{l}\text { latent transforming growth factor beta binding } \\
\text { protein } 1\end{array}$} & \multirow[t]{2}{*}{ Proliferation } & RPS6KA2 & ribosomal protein S6 kinase A2 & No results \\
\hline & & & \multirow{2}{*}{ EPS8 } & epidermal growth factor receptor pathway & \multirow[t]{2}{*}{ Metastasis } \\
\hline COL12A1 & collagen type XII alpha 1 chain & No results & & substrate 8 & \\
\hline PRKCI & protein kinase $\mathrm{C}$ iota & No results & TIMP3 & TIMP metallopeptidase inhibitor 3 & Migration \\
\hline AKT3 & AKT serine/threonine kinase 3 & Cell growth & SPRY2 & sprouty RTK signaling antagonist 2 & Progression \\
\hline IPO5 & importin 5 & No results & LAMB2 & laminin subunit beta 2 & Metastasis \\
\hline EMILIN1 & elastin microfibril interfacer 1 & No results & VDAC1 & voltage dependent anion channel 1 & Metastasis \\
\hline TAF15 & TATA-box binding protein associated factor 15 & No results & ATP6V1D & ATPase $\mathrm{H}+$ transporting $\mathrm{V} 1$ subunit $\mathrm{D}$ & No results \\
\hline CTNNA1 & catenin alpha 1 & Prognosis & MYO1E & myosin IE & No results \\
\hline EFNB2 & ephrin B2 & No results & ACVRL1 & activin A receptor like type 1 & No results \\
\hline ATF4 & activating transcription factor 4 & Apoptosis & SOX9 & SRY-box transcription factor 9 & Proonocic \\
\hline IL1R1 & interleukin 1 receptor type 1 & No results & 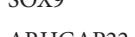 & & Progniosis \\
\hline FRMD6 & FERM domain containing 6 & Metastasis & ARHGAP22 & Rho GTPase activating protein 22 & No results \\
\hline PSAP & Prosaposin & Proliferation & PDGFC & platelet derived growth factor $\mathrm{C}$ & Angiogenesis \\
\hline ITGA2 & integrin subunit alpha 2 & Progression & TNC & tenascin $\mathrm{C}$ & Metastasis \\
\hline PCSK5 & proprotein convertase subtilisin/kexin type 5 & No results & NRP2 & neuropilin 2 & Prognosis \\
\hline RHOJ & ras homolog family member J & No results & TLE3 & TLE family member 3 & No results \\
\hline ITGB5 & integrin subunit beta 5 & Migration & MYLK & myosin light chain kinase & Metastasis \\
\hline \multirow[t]{2}{*}{ CD59 } & \multirow[t]{2}{*}{ CD59 molecule } & \multirow{2}{*}{$\begin{array}{l}\text { Hepatocar- } \\
\text { cinogenesis }\end{array}$} & ABL2 & ABL proto-oncogene 2 & Prognosis \\
\hline & & & COL1A2 & collagen type I alpha 2 chain & Metastasis \\
\hline CSF1 & colony stimulating factor 1 & Metastasis & SMURF2 & SMAD specific E3 ubiquitin protein ligase 2 & No results \\
\hline THY1 & Thy- 1 cell surface antigen & Prognosis & TJP1 & tight junction protein 1 & Prognosis \\
\hline
\end{tabular}

A

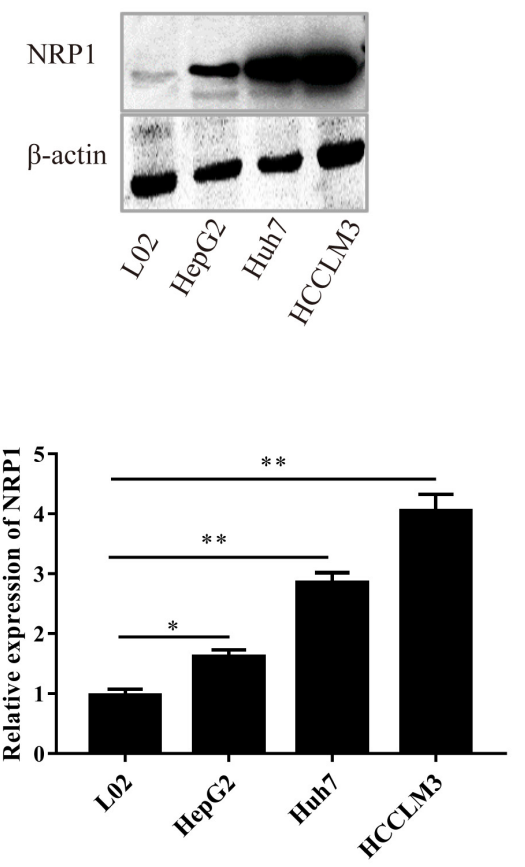

B

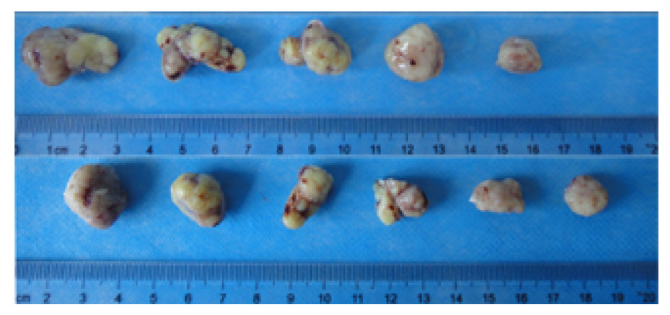

HCCLM3-NC

HCCLM3-shNRP1

$\mathrm{C}$

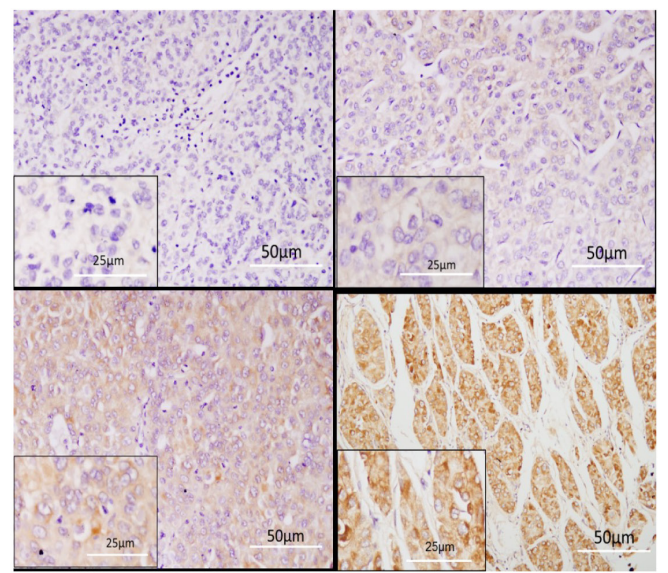

Supplementary Figure S1. A) The level of NRP1 mRNA and NRP1 protein in human L02 hepatocytes and several liver cancer cell lines (HepG2, Huh7, HCCLM3) were detected by qRT-PCR and western blot. Results are shown as means \pm SD. ${ }^{\star} \mathbf{p}<0.05 .{ }^{* *} \mathbf{p}<0.01$. B) Nude mouse subcutaneous tumor tissue when transfected with HCCLM3-NC and HCCLM3-shNRP1 cells. C) The scoring standard of NRP1 expression after immunohistochemical staining. 